\title{
Nova Aquilae 3 (1918).
}

Am Abend des 29. Juni nahm ich am $28^{*}$ Reflektor zwei Aufnahmen von je 20 Minuten, welche die Nova etwa mit der photographischen Helligkeit 9.5 zeigen.

Am 20. und 2 I. Juli versuchte ich eine Spektralaufnahme von zusammen über 5 Stunden Belichtung am Spaltspektrographen des Reflektors unter Verwendung nur eines Prismas. Das Spektrum kam recht schwach.

Es scheint völlig kontinuierlich, ohne helle Bänder. Ob Spuren der Emissionen 436-437 noch eben hervortreten, scheint fraglich; jedenfalls dominiert das kontinuier- liche Band mit der größten Helligkeit etwa bei 440 . Mehr ist nicht mit Sicherheit zu sagen. Mein seit Winter I9 I9-20 nicht mehr versilberter Spiegel ist zu blind.

Zur Festlegung der Helligkeit am 29. Juni maß ich die Durchmesser der Nova und einiger Graffschen Sterne (AN 5054), deren photographische Helligkeiten mir unbekannt sind. Es ergaben sich die Durchmeser: $* 42=245^{\mathrm{p}}, * 35$ $=237, * 34=236$, Nova $=2 I_{5}, * 3 \mathrm{I}=209, * 2 \mathrm{I}=188$, $* 30=187$.

Königstuhl, 1922 Aug. 9.

\section{Mitteilungen über Kleine Planeten.}

Photographic Observations of 945 Barcelona obtained with the Franklin-Adams Star Camera. Magnitude: about I 4.

1922 Gr.m.t. Local sidereal time RA. 1922.0 April I $9.43782 \quad \mathrm{I}_{4}^{\mathrm{h}} \mathrm{III}^{\mathrm{m}} 4 \mathrm{I}^{\mathrm{s}} 7 \quad \mathrm{I}^{\mathrm{h}} \mathrm{III}^{\mathrm{m}} 47^{\mathrm{s}}$. 20.48 I 83 J 5 I 9 1I.5 I 8 II 5 I.88 Nay $\quad$ I.36692 I 3 I $6 \quad 37.9$ I $8 \quad 9 \quad 15.74$ 29.40224 I $5 \quad 58$ I.8 I $7 \quad 3234.26$

$\begin{array}{lllllllll}\text { June } & 4.54478 & 19 & 47 & 29.9 & \text { I } 7 & 20 & 29.40 \text { : }\end{array}$ $\begin{array}{lllllllll}22.28089 & 14 & 37 & 25.9 & \text { I } 6 & 47 & 14.47\end{array}$

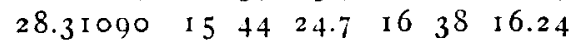

Union Observatory Johannesburg, 1922 June 30 H. E. Wood.

$677 \mathrm{Aaltje}$

Obgleich die Elemente von I 909 (AN I88.23I) durch die Auffindung I $9 \mathrm{I}_{\mathrm{I}}\left(\mathrm{B}-\mathrm{R}=+\mathrm{I}^{\mathrm{m}} \cdot 4+\mathrm{II}^{\prime}\right)$ und $\mathrm{I} 9 \mathrm{I}_{2}(\mathrm{~B}-\mathrm{R}$ $\left.=+2^{\mathrm{m}} \cdot 9+3^{\prime}\right)$ hinreichend bestätigt $\mathrm{zu}$ werden schienen, konnte der Planet I914, 1916, I917, I919 und I92 I nicht aufgefunden werden. Da es durch I)istanzenvariation aus I 909 Febr. I 5 und April io nicht gelang, die Beobachtungen von I 9 I I und I 9 I 2 hinreichend darzustellen, wurde eine Bahn. bestimmung aus I 9 I I Juni 28 , Juli 30 , Aug. 20 allein unternommen, die merklich abweichende Elemente ergab. Sie steliten I 909 rückwärts gut dar $\left(+0^{m} \cdot 9-2^{\prime}\right)$, ergaben aber für IgI2 eine große Abweichung $\left(+24^{\mathrm{m}} \circ \mathrm{o}^{\circ} 25^{\prime}\right)$, die keinen Zweifel ließ, daß der Ort r9 12 Okt. I 2 (B 3045.02, AN I93. I6) nicht zu 677 Aaltje gehörte; damit fanden die wiederholten vergeblichen Versuche, 677 Aaltje aufzufinden, ihre Erklärung. An dem mit der neuen Bahn für 1 9 1 2 Oktober gerechneten Ort gelang es nun Herm Dr. Reinmuth, auf B $3 \circ 37$ (I9 I 2 Okt. 10) das richtige Objekt aufzufinden:

B 3037.04 I 9 I 2 Okt. Io $8^{\mathrm{h}} 26 \mathrm{~m}$.9 Kigt.:

$\mathrm{I}^{\mathrm{h}} 20^{\mathrm{m}} \cdot 5+2 \mathrm{I}^{\circ} 9^{\prime},-\mathrm{I}^{\mathrm{m}} \cdot \mathrm{\circ}-6^{\prime}$, I $3^{\mathrm{m}} \cdot 3,\left(\mathrm{~B}-\mathrm{R}=-0^{\mathrm{m}} \cdot 6-3^{\prime}\right)$.

Damit sind die neuen Elemente hinreichend bestätigt; mit ihnen ergibt sich für die bevorstehende Erscheinung folgende Ephemeride, die vielleicht noch eine kleine negative Korrektion erfordern dürfte $\left(\mathrm{Gr} .13^{\mathrm{m}} \cdot 3\right)$ :

\begin{tabular}{|c|c|c|c|c|c|}
\hline 1922 & $\alpha$ & $\delta$ & 1922 & $x$ & $\delta$ \\
\hline Sept. I 8 & $0^{\mathrm{h}} 41^{\mathrm{m}} \cdot 7$ & $+\mathrm{I} 7^{\circ} 45^{\prime}$ & Okt. I 2 & $\mathrm{O}^{\mathrm{h}} 23^{\mathrm{m}} \cdot 2$ & $+15^{\circ}$ \\
\hline 26 & o 35.6 & $\begin{array}{r}+17 \text { I } 5 \\
+16\end{array}$ & 20 & O 17.6 & +15 \\
\hline kt. $\quad 4$ & 29 & +1637 & 28 & O I 2.8 & I 4 \\
\hline
\end{tabular}

Das fehlerhaft identifizierte Objekt B 3045.02 (I9 I 2 Okt. 12) ist als neu anzusehen und erhält die Bezeichnung I 9 I 2 a.

Berlin-Dahlem, 1922 Aug. I 5. F. Cohn.

Benennungen.

Zur dauernden Erinnerung an die durch Herrn Staatssekretär Hoover organisierte materielle Hilfe Amerikas zugunsten der Bevölkerung Österreichs, insbesondere für Wien und die Mitglieder der Wiener Hochschulen, hat der akademische Senat der Wiener Universität den Planeten 9.32 [ $1920 \mathrm{GV}]$ Hooveria benannt.

F. Palisa.

Prof. 7 . Hartmann hat dem von ihm in La Plata aufgefundenen Planeten r 92 I KT den Namen Angelica beigelegt.

Anzeige. Als Ergänzungshefte zu den Astronomischen Nachrichten sind 6 Abhandlungen erschienen, die von der Expedition zu den nachstehend angegebenen Preisen bezogen werden können. Die Kosten für die Drucklegung dieser Arbeiten konnten bestritten werden aus Mitteln, die der Redaktion von der Notgemeinschaft für die deutsche Wissenschaft und der New York Emergency Society bewilligt wurden, sowie aus einer von einem Freunde der Zeitschrift gestifteten Spende und einem leitrage der Sternwarte zu Sonneberg für die unter Nr. 5 genannte Arbeit.

Die Ergänzungshefte sollen von jetzt ab, um eine bequemere und übersichtlichere Aufbewahrung zu ermöglichen, zu Bänden zusammengefaßt werden. Die früher erschienenen 18 Hefte würden die drei ersten Bände der Reihe bilden. Titelblätter auch für diese Bände werden bei Abschluß des 4. Bandes nachgeliefert werden.

Auch die neu erschienenen Hefte sind von der Fxpedition einzeln zu beziehen zu folgenden Preisen:

I. H. Büttncr. I)ie Bahn des Kometen 1853 III (Klinkerfucs). 3 I S. $4^{\circ}$. . . . . . . . . . . I 6 . M.

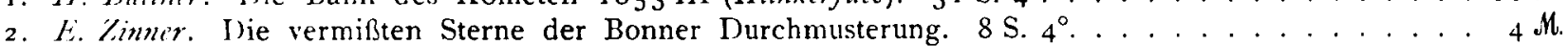

3. E. Zinner. Mitteilungen über veränderliche und verdächtige Sterne. i $8 \mathrm{~S} .4^{\circ}$. . . . . . . . . . г $\mathrm{M}$.

4. H. Büther. Iie Bahn des Kometen von I 779. 1 7 S. $4^{\circ} \ldots \ldots \ldots \ldots$

5. C. Hoffimeister. Entersuchungen zur astronomischen Theorie der Sternschnuppen. 33 S. $4^{\circ}$. . . . I 7 M.

6. H. Osten. Verbesserung von Beckers Theorie von 29 Amphitrite. ${ }_{2} 5$ S. $4^{\circ}$. . . . . . . . . . . . i 3 M. Die Preise für das Auștand sind gemäß dem Verhältnis des Inlandpreises eines Bandes der AN zum Auslandpreise zu berechnen.

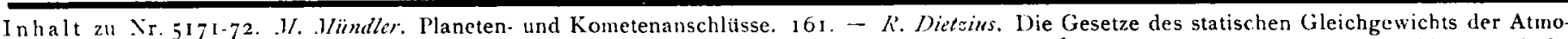
sphären von Himmelskörpern. I81. - H. Osthoff. I)ie Veränderlichkeit von $\alpha$ Orionis. I $87 .-K$. (iraff. Photometrische Beobachtungren. 189. - M.Wolf. Nova Aquilae 3 1918). 191. - Mitteilungen uber Kleine Planeten. 19I. - Anzeige. I91. 